\title{
Multi-body simulation of a human thumb joint by sliding surfaces
}

\author{
Alexander Synek, Marcus Settles, and Georg Stillfried
}

\begin{abstract}
The development of anthropomorphic robotic grippers requires profound knowledge about the functionality of the human hand. For investigating its kinematic and dynamic properties, numerous biomechanical models have been established based on the assumption of fixed rotational axes. Even though this approach has proven to be accurate for most joints of the hand, difficulties have been reported for modelling the movement of the thumb. In order to investigate errors resulting from the thumb carpo-metacarpal joint, a new modelling approach is pursued that is based on contacting surfaces and stabilizing tissues. The joint is modelled as a multi-body system and driven by forces exerted by the cartilage contact, ligaments and muscles. Comparing the simulation results to anatomical literature reveals the capabilities of the proposed approach, but also the necessity of further improvements for its applicability in biomechanical investigations.
\end{abstract}

Keywords: CMC joint, thumb, contact, simulation

\section{INTRODUCTION}

The human hand is essential for everyday interaction with the environment-whether a large object needs to be lifted or a thread has to be inserted into a needle hole. Its versatility is the result of several sophisticated biological mechanisms and has drawn the attention of engineers ever since. Inspired by the human hand, the Institute of Robotics and Mechatronics of the German Aerospace Center (DLR) is developing robotic systems that are capable of performing various tasks in human environments and are intended to be applied in the fields of tele-manipulation, service robotics, and prosthetics.

As a first step towards creating an anthropomorphic robotic gripping system, the human hand needs to be investigated regarding both its kinematic and dynamic properties. A kinematic description of the whole hand has recently been developed based on magnetic resonance imaging (MRI) [1]. The MRI images of several postures were used in order to determine the optimal position and orientation of fixed rotational axes approximating the joint movement. The resulting 24 degrees of freedom (DoF) model has proven to be very accurate, especially regarding the translational error of the bones. The mean error amounts to a maximum of $0.7 \mathrm{~mm}$ at all joints except for the first carpo-metacarpal joint (CMC1 joint, see Fig. 1), where this value is exceeded by approximately $2 \mathrm{~mm}$. This comparatively large error is considered to be caused either by neglecting the movement

A. Synek was with the Institute of Robotics and Mechatronics, German Aerospace Center (DLR) and the Department of Mechatronics and Robotics, UAS Technikum Wien during the research and is now with the Department of Biomedical Engineering, Vienna University of Technology. E-mail: alexander. synek@hotmail.com

M. Settles is with the Klinikum Rechts der Isar, Technische Universität München. E-mail: msettles@tum. de

G. Stillfried is with the Institute of Robotics and Mechatronics, German Aerospace Center (DLR). E-mail: georg.stillfriededlr.de

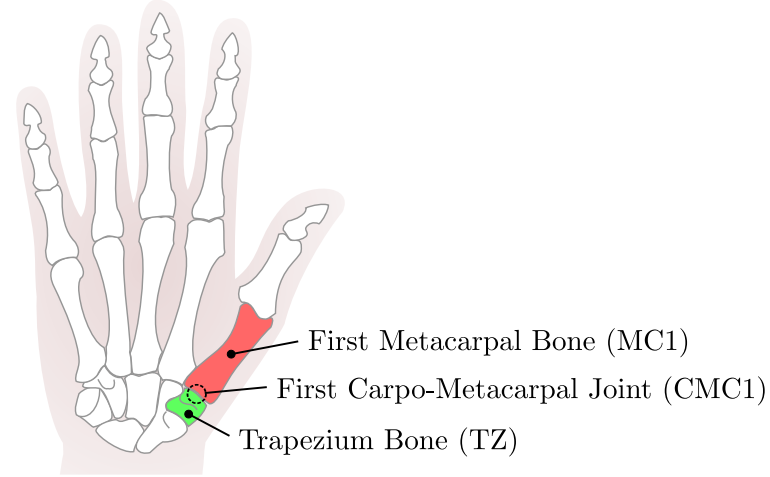

Fig. 1. Location and articulating bones of the first carpo-metacarpal joint

of carpal bones or the general assumption of fixed rotational axes. Problems resulting from present kinematic models of the thumb are also addressed in [2], where it is concluded that alternative kinematic descriptions need to be explored in order to create realistic biomechanical models of the thumb.

In this paper, a new approach of modelling the movement of a human finger joint is presented. Instead of fixed rotational axes, as used in several biomechanical models [3]-[6], the movement is determined based on the actual contacting surfaces and the main stabilizing tissues. The joint is modelled as a multi-body system in the multi-body simulation software SIMPACK (SIMPACK AG, Gilching, Germany) with one fixed and one unconstrained bone, which is driven by the forces of the cartilage in contact, the ligaments and the muscles. Based on the model, the movements resulting from activating single as well as multiple muscles are simulated in time domain. In order to be ultimately able to investigate the origin of the large error in the kinematic model of [1], the modelling and simulating is conducted specifically for the $\mathrm{CMC} 1$ joint.

\section{METHODS AND MATERIALS}

From the anatomical point of view, the main components contributing to joint stability and movement are the articular surfaces, ligaments and muscles [7]. The moving bones are covered by a thin layer of hyaline cartilage at the contacting area and lubricated by synovial fluid. The ligaments consist of highly parallel collagen fibres and are directly attached to adjacent bones. As the attachments move apart, the tissue is stretched and exerts forces restraining the joint motion. Similar to the ligaments, muscles also exert forces as they are passively stretched. Additionally, they can be actively contracted resulting in a controlled movement of the joint.

All mentioned tissues are incorporated in the multi-body 


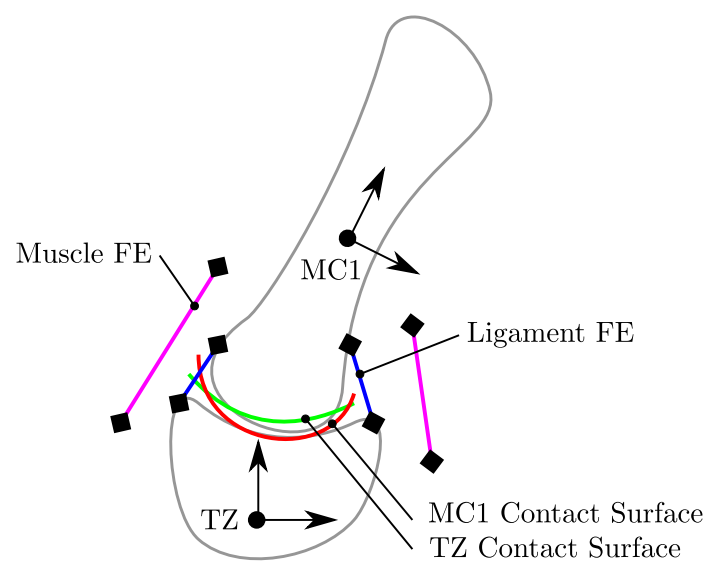

Fig. 2. A schematical depiction of the multi-body system representing the $\mathrm{CMC} 1$ joint. The $\mathrm{MC} 1$ bone is unconstrained and driven by the forces exerted by the contact FE (red/green), ligament FE (blue) and muscle FE (purple).

system representing the $\mathrm{CMC} 1$ joint (see Fig. 2). In this system, the articulating bones are assumed to be rigid and defined by body-fixed coordinate systems and their respective inertial properties. The trapezium bone is fixed whereas the first metacarpal bone is unconstrained in 3D space, hence, possesses 6 DoF. Forces acting upon the unconstrained bone determine its movement and are computed by so called force elements (FE) that represent the cartilage contact, ligaments and muscles. Two non-deformable surfaces form the contact geometry and penetrate in contact. The contact forces and moments acting at the first metacarpal bone are calculated based on the penetration depth and will push the bones apart. This movement is restrained by the ligament and muscle force elements, which exert tensile forces along the connection line of a trapezial and a metacarpal attachment point.

\section{A. Modelling the articular contact}

Articular surfaces highly influence the joint movement. Idealized shapes serve as means of distinguishing between different joint types, such as hinge, ball or condyloid joint. The CMC1 joint is referred to as a saddle joint since its articular surfaces are shaped somewhat like a horse saddle with a slightly curved ridge and a perpendicular groove [8]. In order to take the actual shape of the surfaces into account, the contact geometry is created based on MRI images. MRI is a 3D imaging technique that detects signals of soft tissue exposed to a strong magnetic field and excited by a sequence of radio frequency pulses [9]. Using a T1-weighted fast field echo (T1-FFE) sequence and the principle of selective excitation technique (ProSet), images of the CMC1 joint are recorded that allow to identify the contour separating the bone from the cartilage (see Fig. 3). An anisotropic resolution of $0.99 \times 0.52 \times 0.52 \mathrm{~mm}^{3}$ is obtained within a recording time of approximately $5 \mathrm{~min}$ by utilizing a Philips Achieva MRI scanner with a magnetic field strength of $1.5 \mathrm{~T}$ and a Philips Micro-47 surface coil.

In order to create a contact geometry that represents

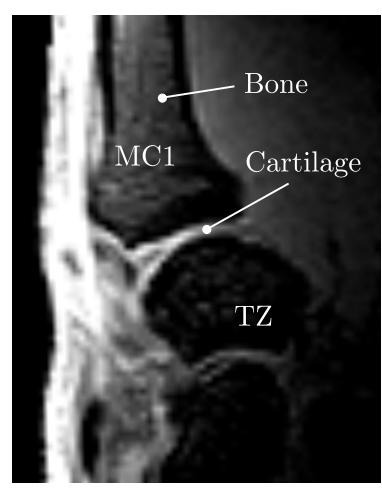

Fig. 3. A slice of the MRI image of the $\mathrm{CMC} 1$ joint, visualizing the bones (dark) and the thin layer of cartilage (bright)

the undeformed articular surfaces, the following steps are conducted: Firstly, the volume of the bones is segmented manually from the MRI images using the 3D Dicom imaging tool Amira (Visage Imaging Inc., Andover, MA, USA). Secondly, a polygonal bone surface consisting of a set of vertices and faces is created in MATLAB (The Mathworks, Natick, MA, USA) by applying box filtering, the marching cubes algorithm and Laplacian smoothing. Thirdly, the bone surface is dilated along the vertex normals by a cartilage thickness of $1 \mathrm{~mm}$, as estimated from the MRI images, and smoothened by an additional iteration of Laplacian smoothing. For qualitative comparison, the surfaces are intersected by a plane coincident with an MRI slice (see Fig. 4).

As can be seen from Fig. 4, the cartilage surfaces are penetrating in contact. In order to compute contact forces depending on the amount of penetration, the polygonal contact model (PCM) [10] is used. PCM is based on the elastic foundation theory, which assumes rigid surfaces to be covered by a layer of springs. For polygonal surfaces, spring elements are created for each face within the contact area. In contact, the springs are deflected and exert forces as defined by a force law. This approach does not take shear forces into account but allows a fast and robust computation even for conforming or multiple contacts with complex contact geometries.

In order to model articular contact, the properties of

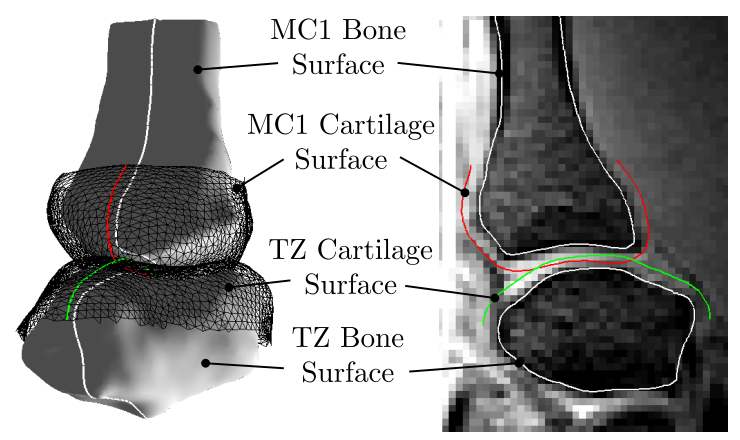

Fig. 4. Polygonal bone and cartilage surfaces of the first metacarpal and trapezium. The red and green lines result from intersecting the surfaces by a plane coincident with the MRI slice displayed on the right 
hyaline cartilage are incorporated by adapting the force law. Even though the cartilage behaves complexly over time, an elastic response is anticipated in case of short loading times [11]. Assuming a contact stiffness of $c=1 \mathrm{~N} / \mathrm{mm}^{3}$ as used in [12] with a stiffness increase as proposed by [13], the force exerted by an individual spring $k$ is calculated as follows:

$$
F_{k}=-c \cdot A_{k} \cdot \ln \left(1-\frac{u_{k}}{2 \cdot h}\right)
$$

The force law takes into account the area $A_{k}$ of the individual polygon in contact and the cartilage thickness $h$, which has already been determined to be $1 \mathrm{~mm}$. For the calculation of the penetration depth $u_{k}$, the penetrating surface is intersected by the reversed face normal $\mathbf{n}_{k, \mathrm{r}}$ of the active polygon, originating at its centroid $C_{k}$ (see Fig. 5). Since the material pairing is quasi identical, the spring element exerts the calculated force in the exact middle of the intersection point $I$ and the centroid $C_{k}$. The total contact force vector $\mathbf{F}$ and moment vector $\mathbf{M}$ are subsequently calculated with respect to the body fixed coordinate system:

$$
\begin{gathered}
\mathbf{F}=\sum_{k} \mathbf{n}_{k, \mathrm{r}} \cdot F_{k}=\sum_{k} \mathbf{F}_{k} \\
\mathbf{M}=\sum_{k}\left(\mathbf{r}_{k}+\mathbf{n}_{k, \mathrm{r}} \cdot \frac{u_{k}}{2}\right) \times \mathbf{F}_{k}
\end{gathered}
$$

Since the friction between articular surfaces is considered to be either very low, ranging from $\mu=0.001$ to $\mu=0.03$ [14], or even totally negligible for modelling the joint motion [12], no frictional forces are calculated for the articular contact in the present model.

\section{B. Modelling the ligaments}

In order to stabilize the joint, several ligaments are attached directly to the articulating bones. Seven ligaments could be identified in [15] surrounding the CMC1 joint: The superficial anterior oblique ligament (sAOL), deep anterior oblique ligament (dAOL), ulnar collateral ligament (UCL), dorsal first intermetacarpal ligament (dIML), palmar first intermetacarpal ligament (pIML), posterior oblique ligament (POL) and the dorsoradial ligament (DRL).

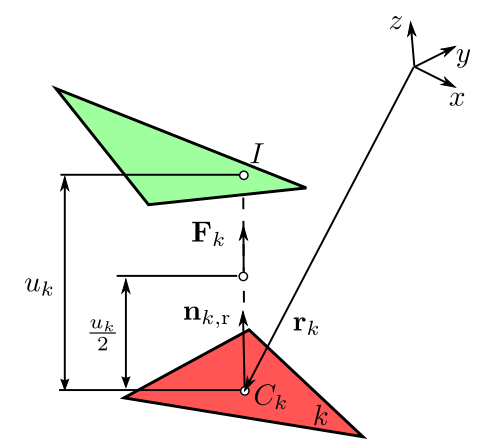

Fig. 5. The principle of the contact force calculation of PCM. A spring element is created for a polygon within the contact area (red) which exerts a force depending on the distance to a polygon of the penetrating surface (green)
Due to the parallel alignment of the strong collagen fibres in the tissue, it is assumed that restraining forces are transmitted along the connection line of the ligament attachments as it is stretched. Hence, the geometry of the force element is reduced to a metacarpal and trapezial attachment point. In order to take the wide insertion areas of the sAOL and the POL into account, they are each modelled as two independent point to point elements. The coordinates of the metacarpal attachments are directly adopted from an anatomical study [15] whereas the trapezial coordinates are estimated based on the respective ligament lengths.

A quadratic force law similar to [3] with a stiffness of $c=7.5 \mathrm{~N} / \mathrm{mm}^{2}$ is used to predict the forces resulting from stretching the ligament:

$$
F= \begin{cases}\frac{C S A}{C S A_{\text {avrg }}} \cdot c \cdot\left(l-l_{0}\right)^{2} & \text { for } l>l_{0} \\ 0 & \text { for } l \leq l_{0}\end{cases}
$$

In order to take the differing tissue architecture into account, the force is additionally scaled using the ligament specific cross sectional area $C S A$ and the averaged cross sectional area $C S A_{\mathrm{avrg}}=7.27 \mathrm{~mm}$ [15]. The initial length $l_{0}$ is determined after the assembly of the whole model and does not necessarily correspond to the distance between the ligament attachment coordinates.

\section{Modelling the muscles}

Muscles do not only actuate the joint but also contribute to the joint stability. Hence, both the active and passive behaviour has to be modelled for each muscle passing the CMC1 joint. Four intrinsic and four extrinsic muscles are incorporated in the present model: The adductor pollicis (AD), abductor pollicis brevis (APB), opponens pollicis (OP), flexor pollicis brevis (FPB), flexor pollicis longus (FPL), extensor pollicis longus (EPL), extensor pollicis brevis (EPB) and the abductor pollicis longus (APL).

Similar to the ligaments, it is assumed that all forces are exerted from a metacarpal to a trapezial attachment point. In [5], the attachment coordinates were determined by means of optimization to experimental data and adopted directly for modelling the muscle force elements. The AD is represented by four elements since it consists of fibre bundles with different functionalities and inserts at a wide area of the metacarpal bone.

Depending on the current strain $\epsilon$ and an activation level $\alpha$, the muscle forces are determined based on a muscle specific Hill model. Neglecting velocity dependent effects, the active force $F_{A}$ is computed as proposed in [16]:

$$
F_{A}=F_{\max } \cdot \alpha \cdot e^{-\left[\frac{(\epsilon+1)\left[0.963+\left(1-1 / i_{a}\right)\right]-1}{0.353 \cdot\left(1-i_{a}\right)}\right]^{2}}
$$

Different muscle architectures are taken into account by using the architecture index $i_{a}$, which is defined as

$$
i_{a}=\frac{l_{f}}{l_{m}}
$$


where $l_{f}$ is the muscle fibre and $l_{m}$ the muscle belly length. The maximum muscle force $F_{\max }$ is calculated based on the physiological cross sectional area (PCSA):

$$
F_{\max }=P C S A \cdot S_{\max }
$$

The muscle maximum stress $S_{\max }$ is set to $0.354 \mathrm{~N} / \mathrm{mm}^{2}$ as used for the simulation of thumb moving muscles in [2]. Values for $i_{a}$ and $P C S A$ are provided in anatomical literature for both intrinsic [17] and extrinsic [18] muscles.

An exponential function is used to predict the muscle passive force $F_{P}$ :

$$
F_{P}=e^{c_{1} \epsilon+c_{2}}
$$

The coefficients $c_{1}$ and $c_{2}$ are determined based on the assumption made in [19] that the muscle exerts $6.5 \%$ of the maximum active force at zero strain and $87 \%$ of the maximum active force at $30 \%$ strain:

$$
\begin{gathered}
c_{2}=\ln \left(0.065 \cdot P C S A \cdot S_{\max }\right) \\
c_{1}=\frac{1}{0.3}\left(\ln \left(0.087 \cdot P C S A \cdot S_{\max }\right)-c_{2}\right)
\end{gathered}
$$

The sum of the active and passive force finally yield the total force exerted by the muscle:

$$
F=F_{A}+F_{P}
$$

Since the tendon stiffness is considered to be large compared to the muscle, it is assumed to be infinitely stiff in the present model.

\section{Assembling the joint model}

In order to arrange all force elements in a physiological fashion, the geometry of the first, second and third metacarpal as well as the trapezium bone are extracted from an MRI image of the whole hand in a reference position. Since the weak signal resulting from the T1-FFE sequence with ProSet requires the use of small coils, different settings have to be applied in order to scan the whole hand. Utilizing a Philips SENSE head coil and a balanced steady-state free precession (b-SSFP) sequence, an interpolated resolution of $0.34 \times 0.38 \times 0.34 \mathrm{~mm}^{3}$ is achieved. The surfaces of the bones are obtained by applying a box filter and the marching cubes algorithm to the volume, followed by three iterations of Laplacian smoothing of the surface.

The vertices of the reference bone surfaces are subsequently used to calculate coordinate systems for the definition of the attachment coordinates of the ligament and muscle force elements. Based on a principal component analysis of the first metacarpal and a hyperboloid fitted to the area of the articular surface of the trapezium, the respective coordinate systems are determined (see Fig. 6).

For arranging the contact geometry, the bone surfaces extracted from the MRI image of the CMC1 joint are matched against the reference bone surfaces using the pose estimation algorithm described in [20]. The algorithm computes the

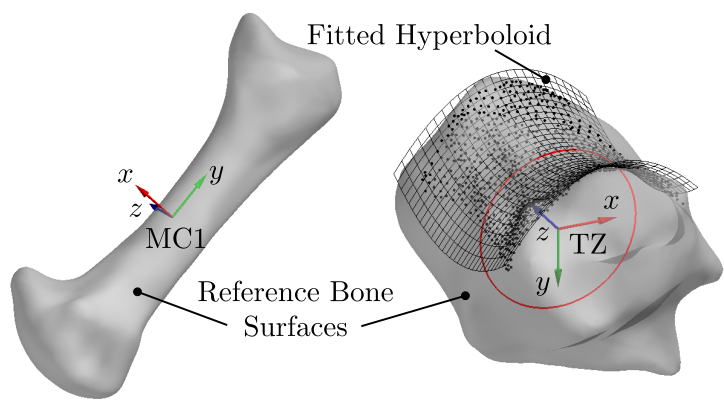

Fig. 6. The coordinate systems of the reference bones, based on a principal component analysis for the first metacarpal and a hyperboloid fitted to the articular surface for the trapezium

rigid motion between a large number of randomly generated congruent triangles of the two sets of vertices and determines the most probable transformation for matching the surfaces. Since the joint posture is different in the MRI images, the transformations of the first metacarpal and the trapezium are determined independently (see Fig. 7).

The inertial properties of the first metacarpal bone are estimated by a cylinder with a diameter of $25 \mathrm{~mm}$ and a height of $50 \mathrm{~mm}$. Assuming a tissue density of $1100 \mathrm{~kg} / \mathrm{m}^{3}$ [3], the mass of the bone amounts to approximately $27 \mathrm{~g}$. The axes of the calculated coordinate system are considered to be coincident with the principal axes of inertia with respective principal moments of inertia of $I_{x x}=I_{z z}=6.68 \mathrm{Nmm}^{2}$ and $I_{y y}=2.11 \mathrm{Nmm}^{2}$.

The complete model of the $\mathrm{CMC1}$ joint consists of eleven muscle force elements, nine ligament force elements, one contact force element and one unconstrained body (see Fig. 8).

\section{E. Determining the initial position}

In order to simulate movements based on the model of the $\mathrm{CMC} 1$ joint, it is necessary to determine an initial position which satisfies the conditions of static equilibrium. For this purpose, the initial lengths and strains of the ligament and muscle force elements are calculated which compensate for the force $\mathbf{F}_{0}$ and moment $\mathbf{M}_{0}$ exerted by the contact force element in the reference position. Based on the vectors $\mathbf{r}_{i}$

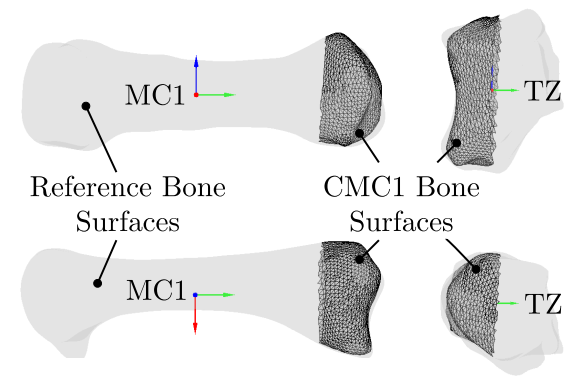

Fig. 7. The results of the pose estimation algorithm for matching the bone surfaces of the MRI image of the CMC1 joint against the bone surfaces of the reference MRI image 


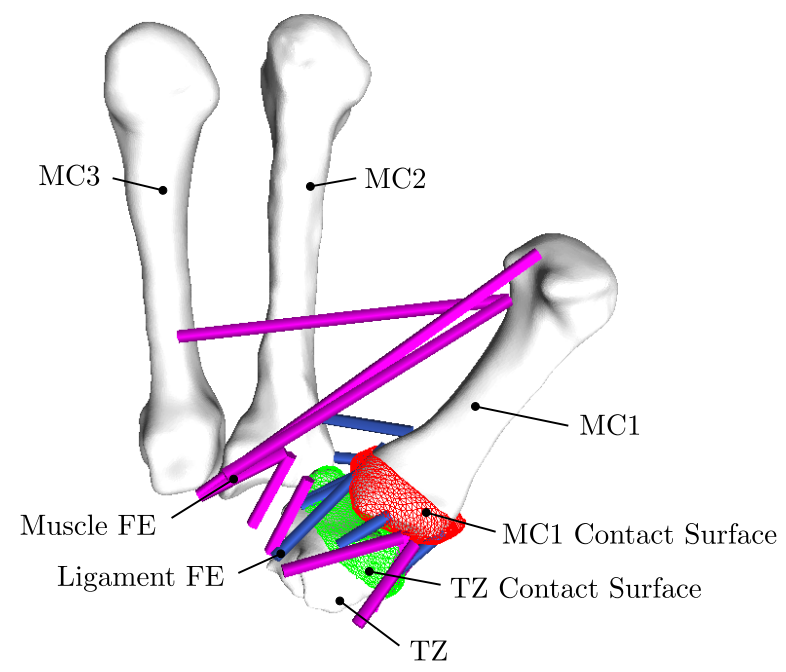

Fig. 8. The complete model of the $\mathrm{CMC} 1$ joint, containing nine ligament force elements (blue), eleven muscle force elements (purple) and one contact force element (red/green)

pointing to the metacarpal attachment and the unit vectors $\mathbf{n}_{i}$ pointing from the metacarpal to the trapezial attachment, an under-determined system of equations is established:

$$
\left[\begin{array}{ccc}
\mathbf{n}_{1} & \cdots & \mathbf{n}_{20} \\
\mathbf{r}_{1} \times \mathbf{n}_{1} & \cdots & \mathbf{r}_{20} \times \mathbf{n}_{20}
\end{array}\right]\left[\begin{array}{c}
F_{1} \\
\vdots \\
F_{20}
\end{array}\right]=\left[\begin{array}{c}
-\mathbf{F}_{\mathbf{0}} \\
-\mathbf{M}_{\mathbf{0}}
\end{array}\right]
$$

The system is solved for the forces $F_{i}$ by conducting a constrained linear least-squares optimization in MATLAB. Initial lengths and strains are obtained by rearranging Equ. 4 and Equ. 8. Subsequently, SIMPACK is used to determine a static equilibrium position which minimizes remaining accelerations caused by the force residual of the constrained optimization.

\section{RESULTS}

Simulations of the movement of the first metacarpal bone are conducted in time domain by activating single or multiple muscles. SIMPACK is used for both establishing and numerically solving the equations of motion. The computation time using the differential algebraic system solver (DASSL) with a tolerance of $5 \cdot 10^{-5}$ and a maximum step size of $10^{-4}$ amounts to approximately $300 \mathrm{~s}$ for a simulation time of $0.5 \mathrm{~s}$.

To be able to compare the simulation results to anatomical literature, the orientation of the first metacarpal coordinate system with respect to the trapezium coordinate system is interpreted in anatomical terms. A rotation around the $x$ axis describes ad-/abduction, around $y$ pro-/supination and around $z$ flexion/extension (see Fig. 6 and 9). We chose $x$ $z-y$ Euler angles so that the ab-/adduction axis is fixed to the base of the hand, and the pro-/supination axis always points in longitudinal direction of the MC1 bone.

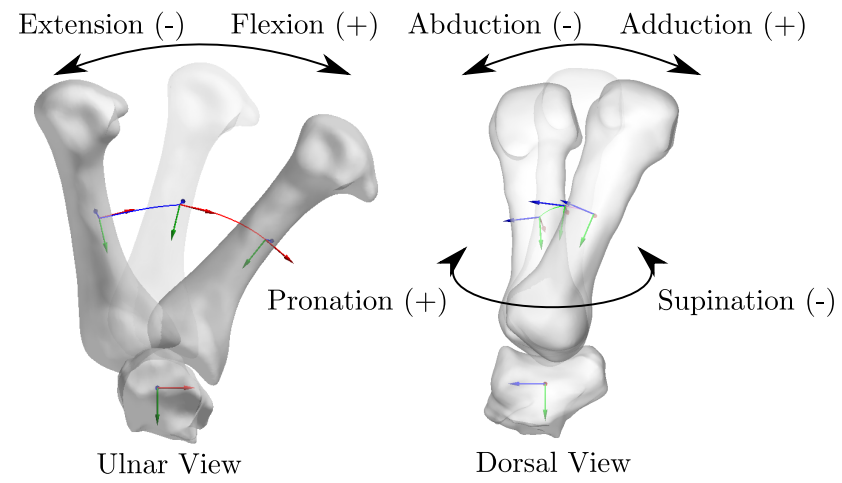

Fig. 9. The anatomical interpretation of the orientation of the first metacarpal coordinate system

\section{A. Single muscle activation}

Each muscle of the model is activated in order to determine its influence on the joint movement. A ramp function is used to increase the activation level $\alpha$ from zero at $t=0.1 \mathrm{~s}$ to the maximum activation $\alpha=1$ with a slope of $3.3 \mathrm{~s}^{-1}$. The result is visualized by plotting the flexion/extension angle against the adduction/abduction and the pronation/supination angle respectively (see Fig. 10).

\section{B. Range of motion}

The range of motion is estimated by activating the muscles considered to be mainly contributing to the flexion, extension, adduction and abduction movement. Similar to the single muscle activation, a ramp function increases the respective activation levels to their maximum within $0.3 \mathrm{~s}$ and a total simulation time of $0.5 \mathrm{~s}$. The maximum flexion of $38.6^{\circ}$ results from activating the $\mathrm{AD}, \mathrm{APB}, \mathrm{FPL}, \mathrm{OP}$ and FPL, the maximum extension amounts to $12.5^{\circ}$ from activating the the EPL, EPB and APL. The APB, OP and APL are activated for the maximum abduction of $3.3^{\circ}$ and the EPL and FPB for the maximum adduction of $23.0^{\circ}$ (see Fig. 11).

\section{DISCUSSION}

Generally, modelling a human joint as a multi-body system has proven to be a fast and simple approach for determining

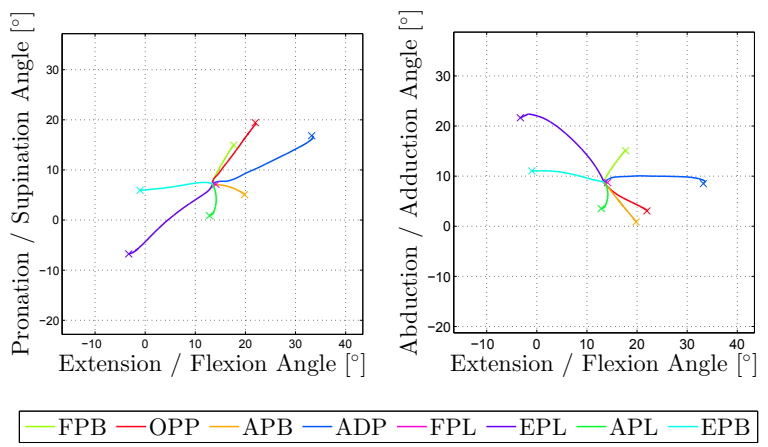

Fig. 10. The results of $0.5 \mathrm{~s}$ simulations of activating single muscles, interpreted in anatomical terms 


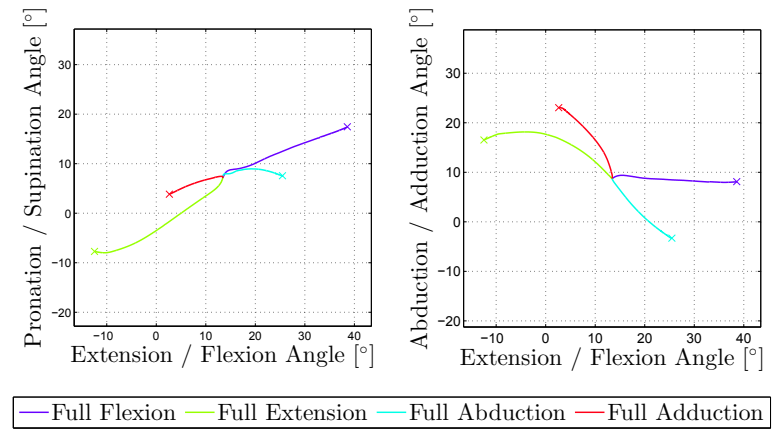

Fig. 11. The results of $0.5 \mathrm{~s}$ simulations of activating multiple muscles in order to estimate the range of motion

the movement based on the actual contact geometry and the main stabilizing tissues. The major drawback is the lack of representative mechanical and geometrical data of the tissues involved, which leads to errors as the data of different sources has to be combined. In order to determine the plausibility of the model of the CMC1 joint, we compared the simulation results to findings in literature.

Quantitative comparison. Our simulation returns a flexion/extension range of $51.0^{\circ}$ and an ab-/adduction range of $26.4^{\circ}$. The true range of motion of the joint (measured from additional MRI images of the joint in its extreme poses) is about $48^{\circ}$ for flexion/extension and about $40^{\circ}$ for ab/adduction. Literature provides values of $50-70^{\circ}$ [21] and $63.4 \pm 9.4^{\circ}$ [22] for flexion/extension and 40-60 [21] and $47.8 \pm 6.3^{\circ}$ [22] for ab-/adduction, respectively.

Qualitative comparison. Furthermore, we compared the simulation results of single muscle activation to anatomical literature [7], [21] and experimental data [23]. Our simulation mostly agrees with the literature except for some details. Consistently, APB, OP and FPL cause flexion and abduction, and EPL causes extension and adduction. While in our simulation, EPB causes extension and adduction, in [21], [23] it causes extension and abduction. FPB causes in our simulation flexion and adduction and in [7], [21], [23] flexion and adduction. The functionality of the AD is considered to be dependent on the current state of the joint [7]. However, the abduction movement caused by the $\mathrm{AD}$ in the initial posture of our model is contradictory to [21] and [23].

As the comparison shows, the present model is capable of simulating physiological movements, but requires further improvements to be applicable in biomechanical investigations. The most promising possibility to improve the model is to use MRI scans of several joint postures in order to find the optimal force element parameters. An improved version of the model of the CMC1 joint could finally be used in kinematic and dynamic hand modelling for determining the influence of single tissues and contacting surfaces on the joint motion, as well as in medical fields such as endoprosthetics.

\section{ACKNOWLEDGMENTS}

This work has been partially funded by the European Commission's Seventh Framework Programme as part of the project The Hand Embodied under grant no. 248587.

\section{REFERENCES}

[1] G. Stillfried and P. van der Smagt, "Movement model of a human hand based on magnetic resonance imaging (MRI)," in International Conference on Applied Bionics and Biomechanics (ICABB), 2010.

[2] F. J. Valero-Cuevas, M. E. Johanson, and J. D. Towles, "Towards a realistic biomechanical model of the thumb: the choice of kinematic description may be more critical than the solution method or the variability/uncertainty of musculoskeletal parameters," Journal of Biomechanics, vol. 36, no. 7, pp. 1019 - 1030, 2003.

[3] J. L. Sancho-Bru, A. Pérez-González, M. Vergara-Monedero, and D. Giurintano, "A 3-d dynamic model of human finger for studying free movements," Journal of Biomechanics, vol. 34, no. 11, pp. 1491$1500,2001$.

[4] N. Brook, J. Mizrahi, M. Shoham, and J. Dayan, "A biomechanical model of index finger dynamics," Medical Engineering \& Physics, vol. 17 , no. 1 , pp. 54-63, 1995.

[5] J. Z. Wu, K.-N. An, R. G. Cutlip, M. E. Andrew, and R. G. Dong, "Modeling of the muscle/tendon excursions and moment arms in the thumb using the commercial software anybody," Journal of Biomechanics, vol. 42, no. 3, pp. 383-388, 2009.

[6] D. J. Giurintano, A. M. Hollister, W. L. Buford, D. E. Thompson, and L. M. Myers, "A virtual five-link model of the thumb," Medical Engineering \& Physics, vol. 17, no. 4, pp. 297-303, 1995.

[7] P. W. Brand and A. M. Hollister, Clinical Mechanics of the Hand, 3rd ed. Missouri: Mosby, Inc., 1999.

[8] K. Kuczynski, "Carpometacarpal joint of the human thumb," Journal of Anatomy, vol. 118, no. Pt 1, pp. 119-126, September 1974.

[9] D. Weishaupt, V. D. Köchli, and B. Marincek, Wie funktioniert MRI?, sixth edition ed. Heidelberg: Springer, 2009.

[10] G. Hippmann, "Modellierung von Kontakten komplex geformter Körper in der Mehrkörperdynamik," Ph.D. dissertation, Technical University of Vienna, October 2004.

[11] R. Hori and L. Mockros, "Indentation tests of human articular cartilage," Journal of Biomechanics, vol. 9, no. 4, pp. 259-268, 1976.

[12] K. An, S. Himeno, H. Tsumura, T. Kawai, and E. Chao, "Pressure distribution on articular surfaces: Application to joint stability evaluation," Journal of Biomechanics, vol. 23, no. 10, pp. 1013-1020, 1990.

[13] L. Blankevoort, J. Kuiper, R. Huiskes, and H. Grootenboer, "Articular contact in a three-dimensional model of the knee," Journal of Biomechanics, vol. 24, no. 11, pp. 1019 - 1031, 1991.

[14] I. C. Clarke, R. Contini, and R. M. Kenedi, "Friction and wear studies of articular cartilage: A scanning electron microscope study," Journal of Lubrication Technology, vol. 97, pp. 358-368, 1975.

[15] M. Nanno, W. L. B. Jr, R. M. Patterson, C. R. Andersen, and S. F. Viegas, "Three-dimensional analysis of the ligamentous attachments of the first carpometacarpal joint," The Journal of Hand Surgery, vol. 31, no. 7, pp. 1160-1170, 2006.

[16] K. Kaufman, K.-N. An, W. Litchy, and E. Chao, "Physiological prediction of muscle forces-I. Theoretical formulation," Neuroscience, vol. 40, no. 3, pp. $781-792,1991$.

[17] M. D. Jacobson, R. Raab, B. M. Fazeli, R. A. Abrams, M. J. Botte, and R. L. Lieber, "Architectural design of the human intrinsic hand muscles," The Journal of Hand Surgery, vol. 17, no. 5, pp. 804-809, 1992.

[18] R. L. Lieber, M. D. Jacobson, B. M. Fazeli, R. A. Abrams, and M. J. Botte, "Architecture of selected muscles of the arm and forearm: Anatomy and implications for tendon transfer," The Journal of Hand Surgery, vol. 17, no. 5, pp. 787 - 798, 1992.

[19] R. D. Woittiez, P. A. Huijing, H. B. K. Boom, and R. H. Rozendal, "A three-dimensional muscle model: A quantified relation between form and function of skeletal muscles," Journal of Morphology, vol. 182, no. 1 , pp. 95-113, 1984.

[20] U. Hillenbrand, "Pose clustering from stereo data." in VISAPP (Workshop on Robot Perception), L. Iocchi and D. G. Sorrenti, Eds. INSTICC - Institute for Systems and Technologies of Information, Control and Communication, 2008, pp. 23-32.

[21] I. A. Kapandji, Funktionelle Anatomie der Gelenke. Stuttgart: Thieme, 2006.

[22] Z.-M. Li and J. Tang, "Coordination of thumb joints during opposition," Journal of Biomechanics, vol. 40, no. 3, pp. 502 - 510, 2007.

[23] K. R. Kaufman, K.-N. An, W. J. Litchy, W. P. Cooney, and E. Y. S. Chao, "In-vivo function of the thumb muscles," Clinical Biomechanics, vol. 14, no. 2, pp. $141-150,1999$. 